\title{
Transient Otoacoustic Emissions and Auditory Brainstem Responses in Low-Risk Cohort of Newborn and One-Month-Old Infants: Assessment of Infant Auditory System Physiology in the Prenatal Alcohol in SIDS and Stillbirth Network Safe Passage Study
}

DOI: $10.3766 /$ jaaa. 17043

\author{
Yvonne S. Sininger* $\dagger$ \\ Carmen G. Condon \\ Howard J. Hoffman§ \\ Amy J. Elliott $\mid \|$ \\ Hein J. Odendaal** \\ Larry L. Burd $\dagger+\ddagger \S \S$ \\ Michael M. Myers† $\uparrow \uparrow||||$ \\ William P. Fifer $\dagger \uparrow||\|\|$ \\ PASS Network
}

\begin{abstract}
Background: The Prenatal Alcohol and Sudden Infant Death Syndrome and Stillbirth Network, known as the "Safe Passage Study," enrolled approximately 12,000 pregnant women from the United States and South Africa and followed the development of their babies through pregnancy and the infant's first year of life to investigate the role of prenatal alcohol exposure in the risk for sudden infant death syndrome (SIDS) and adverse pregnancy outcomes, such as stillbirth and fetal alcohol spectrum disorders.

Purpose: Auditory system tests were included in the physiologic test battery used to study the effects of prenatal alcohol exposure on neurophysiology and neurodevelopment, as well as potential causal relationships between neurodevelopmental disorders and SIDS and/or stillbirth. The purpose of this manuscript is to describe normative results when using the auditory test battery applied.

Research Design: The test battery included the auditory brainstem response (ABR) and transientevoked otoacoustic emissions (TEOAEs). Data were collected on individual ears of newborns and 1-month-old infants.

Study Sample: From a cohort of 6,070 with auditory system exams, a normative subsample of 325 infants were selected who were not exposed prenatally to alcohol, cigarette smoke, or drugs nor were they preterm or low birthweight. The subsample is small relative to the overall study because of strict criteria for no exposure to substances known to be associated with SIDS or stillbirth and the exclusion of preterm and low birthweight infants. Expectant mothers were recruited from general maternity at two comprehensive clinical sites, in the northern plains in the United States and in Cape Town, South Africa. These populations were selected for study because both were known to be at high-risk for SIDS and stillbirth.

Data Collection and Analysis: ABR and TEOAE recordings were stored electronically. Peak latency and amplitude analysis of ABRs were determined by study personnel, and results were evaluated for differences by age, sex, test site, race, and ear (left versus right).
\end{abstract}

*Department of Head \& Neck Surgery, David Geffen School of Medicine, University of California Los Angeles, Los Angeles, CA; †C\&Y Consultants, LLC, Santa Fe, NM; †Division of Developmental Neuroscience, New York State Psychiatric Institute, New York, NY; §Epidemiology and Statistics Program, National Institute on Deafness and Other Communication Disorders (NIDCD), National Institutes of Health (NIH), Bethesda, MD; 9 Center for Health Outcomes and Population Research, Sanford Research, Sioux Falls, SD; ||Department of Pediatrics, Sanford School of Medicine, University of South Dakota, Sioux Falls, SD; ${ }^{\star \star}$ Department of Obstetrics and Gynaecology, Faculty of Medicine and Health Science, Stellenbosch University, Cape Town, South Africa; ††Department of Pediatrics, University of North Dakota Fetal Alcohol Syndrome Center, Grand Forks, ND; +tUniversity of North Dakota School of Medicine and Health Sciences, Grand Forks, ND; §§University of North Dakota School of Medicine, Grand Forks, ND; 9 ฯDepartment of Psychiatry, Columbia University Medical Center, New York, NY; ||||Department of Pediatrics, Columbia University Medical Center, New York, NY

Corresponding author: Yvonne S. Sininger, Santa Fe, NM 87506; Email: ysininger@cnyconsult.com 
Results: TEOAE findings were consistent with existing literature including the increase in signal-to-noise (SNR) over the first month of life. The SNR increase is due to an increase in amplitude of the emission. TEOAE amplitude asymmetry favoring the right ear was found, whereas SNR asymmetry was not, perhaps because of the small sample size. A nonsignificant trend toward larger responses in female babies was found; a result that is generally statistically significant in studies with larger samples. Latencies were found to be shorter in ABRs elicited in the right ear with amplitudes that were slightly bigger on average. An expected decrease in wave $\mathrm{V}$ latency was observed from birth to 1-month of age, but the finding was of borderline significance $(p=0.058)$.

Conclusions: One month is a short time to judge development of the auditory system; however, the ABR and TEOAE findings were consistent with current literature. We conclude that the auditory system data acquired for the Safe Passage Study, as reflected in the data obtained from this cohort of "unexposed" infants, is consistent with published reports of these auditory system measures in the general population.

Key Words: auditory brainstem response, sudden infant death syndrome, transient-evoked otoacoustic emissions

\begin{abstract}
Abbreviations: $\mathrm{ABR}=$ auditory brainstem response; ANOVA = analysis of variance; $\mathrm{EEG}=$ electroencephalogram or brain wave activity; NP = northern plains sites in North and South Dakota; OME = otitis media with effusion; PASS = the Prenatal Alcohol in Sudden Infant Death Syndrome and Stillbirth; SA = South Africa clinical site; SIDS = sudden infant death syndrome; SNR = signalto-noise ratio; TEOAE = transient-evoked otoacoustic emissions
\end{abstract}

\section{INTRODUCTION}

$\mathrm{T}$ he Prenatal Alcohol in Sudden Infant Death Syndrome and Stillbirth (PASS) Network was established under cooperative agreements with National Institute of Child Health and Human Development, National Institute on Alcohol Abuse and Alcoholism, and National Institute on Deafness and Other Communication Disorders to conduct the Safe Passage Study. This study investigated populations with high prevalence of maternal prenatal alcohol exposure in Cape Town, South Africa (SA; Tygerberg Hospital) and in the northern plains of the United States (NP, Sanford Research, Sioux Falls, $\mathrm{SD})$. These sites allowed the inclusion of three racial populations, including South African mixed-race ancestry, American Indian/Alaskan Native, and Caucasian (Dukes et al, 2014). This large prospective study followed motherbaby dyads from pregnancy through one postnatal year to investigate the hypothesis that prenatal alcohol exposure increases the risk for sudden infant death syndrome (SIDS), stillbirth, and adverse neurodevelopmental outcomes. SIDS is defined as the death of an infant under 1 year of age, usually during sleep, that remains unexplained after complete investigation including autopsy, examination of the death scene, and clinical history review (Willinger et al, 1991).

An important part of the Safe Passage Study involved secondary aims regarding the effects of prenatal alcohol exposure on neurophysiology and neurodevelopment, as well as potential causal relationships between neurodevelopmental disorders and SIDS and/or stillbirth. Protocols were developed and administered by the Columbia University Physiology Assessment Center for a wide variety of physiological assessments designed to evaluate a range of neural functions. These included measures of heart and respiratory rate, blood pressure, fetal movement, cortical [electroencephalogram (EEG)] activity, and auditory system function.

A measure of auditory nerve and brainstem function, the auditory brainstem response (ABR), and a measure of cochlear (outer) hair cell function, transient-evoked otoacoustic emissions (TEOAEs), comprised the auditory system assessments in the Safe Passage Study. These measures are objective, noninvasive, and have been easily and reliably obtained on thousands of infants for clinical and research purposes and used routinely for newborn hearing screening.

The TEOAE is a low-level sound produced by outer hair cells of the cochlea as the result of the amplification they provide during the transduction process when sound (vibration) is changed into a neural impulse. By analyzing the frequency composition of the emission, assessments can be made about the function of the outer hair cells from the various frequency regions of the cochlea. TEOAEs are measures of basic cochlear function but are also modulated by the medial olivo-cochlear efferent system and, therefore, TEOAE status can also reflect brainstem function.

The ABR is a far-field measure of auditory system neural potentials from the auditory nerve and brainstem. The poststimulus latencies and amplitudes of the peaks of the ABR are widely used as an indicator of auditory system development in infants and have been shown to be highly sensitive to subtle dysfunction (Cone-Wesson, 2005).

Children with prenatal exposures and birth factors such as those being followed in the Safe Passage Study have been shown to be at risk for a variety of hearing and communication disorders (Cone-Wesson, 2005). In addition, prenatal exposures including alcohol (Church and Holloway, 1984; Church, 1987) and cigarette smoke (Kable et al, 2009) have been shown to affect auditory 
Table 1. Numbers of Infants by Selected Characteristics Used in Assessment of Auditory Physiological Function

\begin{tabular}{|c|c|c|c|}
\hline & & TEOAE Infants & ABR Infants \\
\hline$\overline{A l l}$ & Number & 296 & 272 \\
\hline \multirow[t]{2}{*}{ Sex } & Female & 167 & 150 \\
\hline & Male & 129 & 122 \\
\hline \multirow[t]{3}{*}{ Race } & American Indian/Alaska Native & 28 & 16 \\
\hline & South African (mixed ancestry) & 200 & 195 \\
\hline & White & 68 & 61 \\
\hline \multirow[t]{2}{*}{ Assessment age } & Newborn & 215 & 215 \\
\hline & 1 month & 81 & 57 \\
\hline \multirow[t]{2}{*}{ Delivery mode } & Caesarean & 32 & 36 \\
\hline & Vaginal & 264 & 236 \\
\hline \multirow[t]{2}{*}{ Birth weight (grams) } & Mean & 3,309 & 3,292 \\
\hline & SD & 457 & 449 \\
\hline \multirow[t]{2}{*}{ Gestational age (weeks) } & Mean & 39.4 & 39.4 \\
\hline & SD & 1.5 & 1.4 \\
\hline
\end{tabular}

Note: All were full-term infants found to be "exposure free" as described in the text.

brainstem development in ways that can be detected with the ABR.

Abnormalities in neonatal TEOAE measures have been shown, in one study, to be predictive of SIDS (Rubens et al, 2008). In particular, the TEOAE response from the right but not the left ear of infants who died of SIDS was significantly smaller than the response found in living matched controls. Sininger and Cone-Wesson (2004) demonstrated that the TEOAE from the right ear of infants is expected to be larger than the response from the left. This right-ear amplitude advantage for TEOAEs, which was absent in the SIDS participants in the Rubens study, is likely the result of asymmetrical outer hair cell modulation by the medial olivo-cochlear system in the brainstem (Sininger and Cone-Wesson, 2004). For that reason, symmetry in the TEOAE amplitude could be an important metric with functional significance for SIDS.

\section{Middle Ear Anomalies and Otitis Media}

The level of the stimulus delivered to the cochlea can be attenuated by conditions of the middle ear such as otitis media with effusion (OME) that is, an ear infection with fluid in the middle ear space or even the presence of naturally occurring substances such as amniotic fluid in the early hours following birth. Fluid in the middle ear space as well as reduced tympanic membrane compliance can attenuate the stimulus reaching the cochlea by as much as $30 \mathrm{~dB}$. Depending on the amount of attenuation, ABR peak amplitudes may be decreased and absolute latencies increased under these conditions. Interpeak latencies, however, are relatively unaffected by stimulus level. OME and middle ear conditions, however, have more pronounced influence on TEOAE results than ABR because both the eliciting stimulus level and the energy of the TEOAE itself are dampened as they are conducted through the middle ear mechanism. Boone et al (2005) found that $64.5 \%$ of infants in their study who failed TEOAE hearing screening had evidence of OME whereas only $0.01-0.03 \%$ were expected to have sensory hearing loss.

A subgroup of infants in the Safe Passage Study were expected to demonstrate OME or at least residual middle ear fluid. Infants who are exposed to cigarette smoke also have been found to have higher prevalence of $\mathrm{OME}$ (Ey et al, 1995). Fortunately, the time frame for evaluating infants in the Safe Passage Study, at birth and at 1 month of age, precedes the peak period for otitis media (Hunter et al, 2007). If OME was present, we expected to see increases in peak latencies and amplitude reductions in the ABRs. However, the more sensitive test is the TEOAE. A higher prevalence of OME has been found in American Indian/Native Alaskan infants relative to the general population (Curns et al, 2002). Hunter et al (2007) found that the TEOAE test was failed in a

Table 2. Maternal Race by Test Site

\begin{tabular}{lrrrrrrr}
\hline & \multicolumn{9}{c}{ Site } & & \\
\cline { 2 - 6 } Maternal Race & SA & NP1 & NP2 & NP3 & NP4 & NP5 & Total (\%) \\
\hline American Indian/Alaska Native & 0 & 0 & 9 & 17 & 2 & 3 & $31(9.5)$ \\
South African (mixed ancestry) & 222 & 0 & 0 & 0 & 0 & 0 & $222(68.3)$ \\
White & 0 & 15 & 0 & 25 & 32 & 0 & $72(22.2)$ \\
\hline
\end{tabular}

Note: SA is the South African site and NP1-NP5 are sites from the northern plains of the United States. 
significant proportion of Native American Infants with OME. If a relationship is found between TEOAE results and ABR latency and/or amplitude results, then the data from individual ears of any child found to have ABRs with increased peak latency or decreased amplitude and an absent TEOAE response in the same ear could be labeled as "possible OME" and analyzed as a separate group. It should be noted that unilateral OME can be present and would not preclude the use of the ABR and TEOAE data from the other ear. Contamination of auditory system measures by OME was expected to be seen in children with good and poor outcomes and therefore should not bias the measurements with regard to prediction of outcome.

This manuscript describes auditory system function from a subsample of infants who were without prenatal exposure to alcohol, cigarette smoke, or drugs of abuse and were otherwise considered healthy babies (e.g., not preterm or low birthweight). The data demonstrate the validity of the Safe Passage Study methodology and provide a normative guideline by which to judge, in future analyses, abnormalities in participants with prenatal exposures.

\section{METHODS}

\section{Data Collection Sites}

Newborn infants were tested 12-96 hours after birth. In the northern plains sites in North and South Dakota (referred to as NP1- NP5), these tests occurred before discharge from the hospital. Because of the rapid $(<24 \mathrm{~h})$ discharge policy at the Cape Town, South Africa clinical site (referred to as $\mathrm{SA}$ ), infants at this site were brought back to the Safe Passage Study testing facilities at either the Tygerberg or Karl Bremer Hospitals 48-96 hours after birth for their newborn assessments. The infants were assessed again at 1 month ( $28 \pm 7$ days) corrected age.

\section{Participants}

Of the cohort of 6,070 infants who received auditory system tests in the Safe Passage Study, 325 were determined to be free of "exposures," with normal gestations and birth weight. This subset of participants had completed 296 TEOAE evaluations and/or 272 ABR tests.

The "exposure-free" infants were singletons born 37-41 weeks postmenstrual age with birth weights $\geq 2,500 \mathrm{~g}$. None of these infants required resuscitation at birth and none were admitted to the neonatal intensive care unit for any reason. Prenatal alcohol exposure information was obtained using a modification of the timeline followback interview (Dukes et al, 2014). Mothers of these infants reported no alcohol consumption, no smoking, and no drugs of abuse during pregnancy. In addition, no evidence of diabetes, hypertension, or preeclampsia during
TEOAEs by ear, age, and pass status

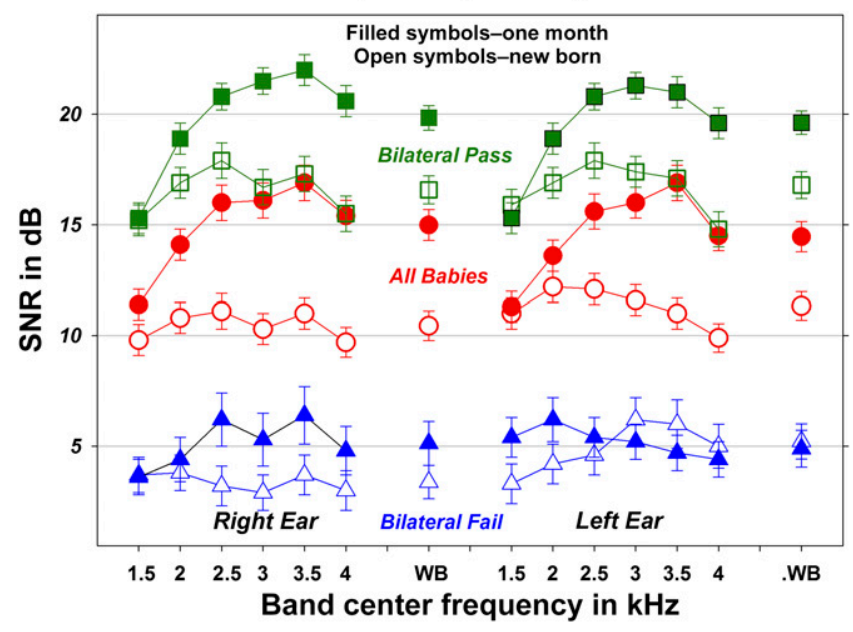

Figure 1. Mean narrow band SNRs of transient OAEs are shown with \pm 1 SE bars. Wideband responses for each ear and condition appear to the right of the frequency-specific data. TEOAE data from all infants appear in the middle of the graph and are depicted in red. Infants evaluated in the newborn period have open symbols and those evaluated at 1 month are shown as filled symbols. As expected, the subset of infants referred to as "bilateral pass" (shown in green) have larger SNRs and those with bilateral fail ratings (shown in blue) have the lowest TEOAE SNRs.

pregnancy was found in the medical records of these mothers. Other demographic information for participants can be found in Table 1.

Auditory assessments made up a portion of the physiologic assessments attempted during the neonatal period. Another physiologic assessment was conducted at 1 month ( \pm 7 days) postnatal age. ABR was attempted at the newborn period and, if missed, was performed at the 1 month assessment. TEOAE, which generally requires less time to administer, was attempted at both assessment times. In all, there were $249 \mathrm{ABR}$ measures and 409 TEOAEs from the left ear and 246 ABRs and 400

Table 3. Distribution of Infants and TEOAE Tests by Selected Characteristics for Infants Who Achieved the "Bilateral Pass" Status on TEOAEs (See Text)

\begin{tabular}{llrc}
\hline & & Infants & TEOAE Tests \\
\hline Age at test & 1 Month & 55 & 128 \\
& Newborn & 113 & 113 \\
Infant sex & Female & 93 & 131 \\
Site & Male & 75 & 110 \\
& SA & 102 & 156 \\
& NP1 & 12 & 16 \\
& NP2 & 6 & 7 \\
& NP3 & 25 & 37 \\
Total & NP4 & 21 & 23 \\
& NP5 & 2 & 2 \\
& & 168 & 241
\end{tabular}

Note: These infants were analyzed separately and assumed to be free of middle ear conditions that could contaminate the results. 
Table 4. ANOVA Results of Variables with Potentially Significant Influence on the TEOAE SNR, Based on All Participants Regardless of "Pass Status"

\begin{tabular}{|c|c|c|c|c|c|}
\hline Source & Type III Sum of Squares & $\mathrm{df}$ & Mean Square & $F$ & Significance, $p$ Values \\
\hline Corrected model & $4,520.42$ & 21 & 215.26 & 3.22 & $<0.001$ \\
\hline Intercept & $4,265.49$ & 1 & $4,265.50$ & 63.78 & $<0.001$ \\
\hline Number of tests & 85.02 & 1 & 85.02 & 1.27 & 0.26 \\
\hline Age & 563.02 & 1 & 563.02 & 8.42 & 0.004 \\
\hline Site & $1,763.60$ & 5 & 352.72 & 5.27 & $<0.001$ \\
\hline Sex & 11.32 & 1 & 11.33 & 0.17 & 0.68 \\
\hline Age * Site & 530.42 & 4 & 132.61 & 1.98 & 0.10 \\
\hline Age * Sex & 17.17 & 1 & 17.18 & 0.26 & 0.61 \\
\hline Sex * Site & 465.56 & 4 & 116.39 & 1.74 & 0.14 \\
\hline Age * Sex * Site & 12.58 & 4 & 3.14 & 0.05 & 0.996 \\
\hline
\end{tabular}

Note: Statistical significance was found only for age (newborn versus 1 month) and test site.

TEOAEs from the right ear from the "exposure-free" cohort.

Infants were enrolled from six sites, five from the two northern plains states in the United States (referred to as NP1-NP5) and one from Cape Town, South Africa (referred to as SA). It is important to note that the distribution of maternal race varied by site. The distribution of infants by mother's race is found in Table 2 .

\section{TEOAE}

Transient-evoked otoacoustic emissions (TEAOEs) were elicited using the Interacoustics, Denmark Otoread, a handheld measurement system appropriate for use with infants. The stimulus for the TEOAE was a broad-band click. The stimulus level was monitored by the microphone in the ear canal probe assembly. The monitored stimulus level was $83.25 \mathrm{~dB}$ SPL in the right ear and $83.23 \mathrm{SPL}$ in the left with standard deviations of 1.03 and $1.08 \mathrm{~dB}$, respectively. The ear probe is comprised of an input portal connected to a transducer to deliver the stimulus and a port connected to a microphone that measures the response. The probe is sealed into the infant's ear with a flexible plastic ear piece. Probe fit in the infant ear canal was monitored using a visual inspection of the spectrum of the stimulus in the ear canal before testing. Automatic feedback was provided to the tester to adjust and optimize individual probe fits.

In previous studies, TEOAEs have been acquired in a manner that stops the averaging when a criterion signalto-noise ratio (SNR) is reached or, if the SNR is not achieved, after a fixed number of sweeps. The current study protocol did not stop based on the SNR; instead, averaged responses were obtained using a fixed number of sweeps in each ear. This allows for comparison of both response amplitude and background noise across ears with all conditions having a balanced opportunity to reduce noise (Sininger and Cone, 2008). Responses were averaged using 300 artifact-free stimulus presentations. The responses from split buffers were compared by correlation to determine the reliability of the recording and, subsequently, re-added to determine response (signal) strength. The two buffers are subtracted to determine the amount of residual noise in the recording. Both the signal and noise were subjected to a Fourier analysis and segmented into six frequency bands with center frequencies of $1.5,2.0,2.5,3.0,3.5$, and $4.0 \mathrm{kHz}$. The SNR was determined for the response as a whole and for each of the individual frequency bands.

\section{ABR}

ABRs were recorded using Intelligent Hearing Systems USBLite $^{\circledR}$. Stimuli consisted of $80 \mathrm{~dB}$ nHL (normalized hearing level) clicks generated by $100 \mu$ sec, alternatingpolarity pulses and presented via Eytmotic ER-3 earphones at a rate of $13 / \mathrm{sec}$. Responses were recorded with stimuli presented individually to each ear. Split-half averages were available to view the response from condensation and rarefaction stimuli in separate buffers. The noninverting electrode was placed at the high-forehead midline with the inverting electrode on the ipsilateral mastoid and the ground electrode on the contralateral mastoid. EEG activity was filtered from 30 to $1500 \mathrm{~Hz}$ at $6 \mathrm{~dB}$ /octave. Twelve millisecond epochs of artifact-free EEG were collected in response to 1,024 stimuli for each of two averages. Sweeps with peak amplitudes $>20 \mu \mathrm{V}$ were rejected without contributing to the average. Two averages from each ear were collected and added for analysis when appropriate. Infants were tested during

Table 5. $p$ Values for Post Hoc Site Differences in Table 4

\begin{tabular}{lccccc}
\hline & SA & NP1 & NP2 & NP3 & NP4 \\
\hline SA & & & & & \\
NP1 & 0.001 & & & & \\
NP2 & 0.807 & 0.035 & & & \\
NP3 & 0.008 & 0.202 & 0.160 & & \\
NP4 & 0.007 & 0.427 & 0.104 & 0.643 & \\
\hline
\end{tabular}

Note: The SA site was found to be significantly different from all other sites with the exception of NP2. 
Table 6. ANOVA Results of Variables with Potentially Significant Influence on the TEOAE SNR, Based on Infants in the "Bilateral Pass" Category

\begin{tabular}{|c|c|c|c|c|c|}
\hline \multicolumn{6}{|c|}{ ANOVA-Dependent Variable: TEOAE SNR } \\
\hline Source & Type III Sum of Squares & df & Mean Square & $F$ & Significance, $p$ Values \\
\hline Corrected model & $1,729.05^{\star}$ & 20 & 86.45 & 3.54 & $<0.001$ \\
\hline Intercept & $4,842.95$ & 1 & $4,842.95$ & 198.07 & $<0.001$ \\
\hline Number of tests & 122.47 & 1 & 122.47 & 5.01 & 0.03 \\
\hline Age & 34.79 & 1 & 34.79 & 1.42 & 0.23 \\
\hline Sex & 17.88 & 1 & 17.88 & 0.73 & 0.39 \\
\hline Site & 588.54 & 5 & 117.71 & 4.81 & $<0.001$ \\
\hline Age * Sex & 4.50 & 1 & 4.50 & 0.18 & 0.67 \\
\hline Age * Site & 146.61 & 4 & 36.65 & 1.50 & 0.20 \\
\hline Sex * Site & 203.50 & 4 & 50.88 & 2.08 & 0.08 \\
\hline Age * Sex * Site & 30.76 & 3 & 10.26 & 0.42 & 0.74 \\
\hline
\end{tabular}

Note: Statistical significance was found only for test site $(p<0.001)$.

natural sleep. The skin was lightly cleaned in preparation for self-adhesive, gelled electrodes. The infant was swaddled and soothed until sleep was achieved.

Each waveform was inspected for typical morphology with the expectation of seeing between three and five distinct peaks. If necessary, split-half waveforms were evaluated to determine response quality, and waveforms were filtered with $100 \mathrm{~Hz}$ high pass filter to aid response visualization. The poststimulus latency and peak-tofollowing-trough amplitude of peaks I, III, and V were labeled by a trained observer and interpeak latencies were computed. Any responses with missing peaks or latencies outside the expected range were examined and verified by the first author.

\section{Statistical Analyses}

A number of factors can influence the SNR of the TEOAE as well as the amplitudes and latencies of the three major peaks of the ABR. The effects of age (newborn versus 1 month), sex, and test site or maternal race were evaluated using analysis of variance (ANOVA). A repeated measures paradigm was applied to the ABR because three peaks in the waveform of the response were evaluated simultaneously. ABR ANOVAs were performed separately for peak latencies and peak amplitudes. The TEOAE was first evaluated for all participants and, subsequently, subdivided to analyze the data from those infants who demonstrated a clear TEOAE response by standard screening criteria (for "pass"). This subset was used to eliminate the potential for noise in the data from the infants who had no clear response.

Effects of factors that might influence the pass/fail dichotomy of the TEOAE were analyzed using the chisquared test for independence. The factors examined were test site, age (newborn versus 1-month old), and sex.

Two approaches were employed to evaluate ear differences (laterality) for TEOAEs and ABR. For TEOAEs, an asymmetry index was created by subtracting the left ear
SNR from the right. This value was subjected to ANOVA to evaluate the same factors and interactions mentioned previously. A common measure of laterality uses a paired $t$-test to evaluate the mean difference in results obtained from both ears of participants. The fixed-number-ofsweeps approach employed in the TEOAE acquisition allowed for separate laterality analysis by paired $t$-test for background noise, amplitude, and SNR of the TEOAE data. This analysis was applied to the group of participants who "passed" a standard TEOAE screening test. The same was applied to the three individual latencies and amplitude measures of the ABR.

Lateral asymmetry, when found in the click-evoked ABR of infants, has consistently favored the right ear by showing shorter latencies and/or larger amplitudes (Eldredge and Salamy, 1996; Sininger et al, 1998; Sininger and Cone-Wesson, 2006). The right ear has also been shown to produce a greater TEOAE SNR than the left (Aidan et al, 1997; Khalfa et al, 1997; Thornton et al, 2003; Saitoh et al, 2006; Sininger and Cone-Wesson,

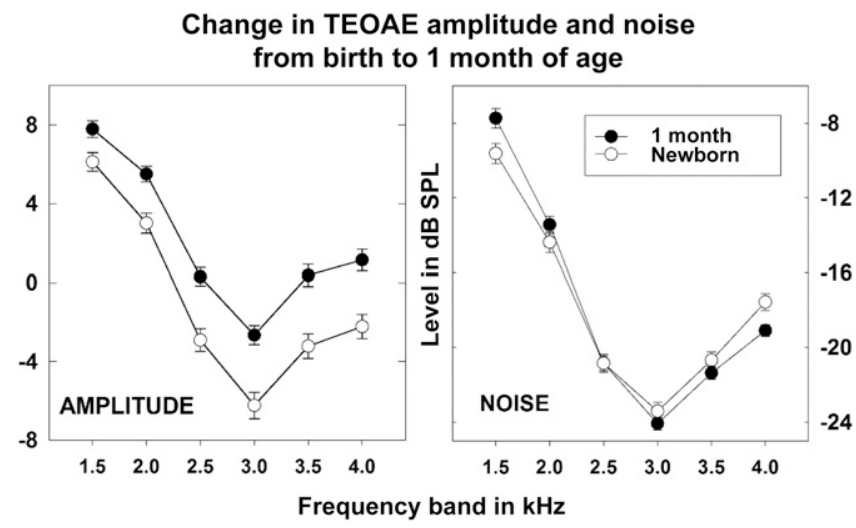

Figure 2. TEOAE amplitude and noise from the "bilateral pass" group, after averaging left and right ears, plotted by infant's age. Based on ANOVA, the change in amplitude with age was significant at $p<0.001$, whereas the effect of age on noise was nonsignificant, $p=0.99$. 


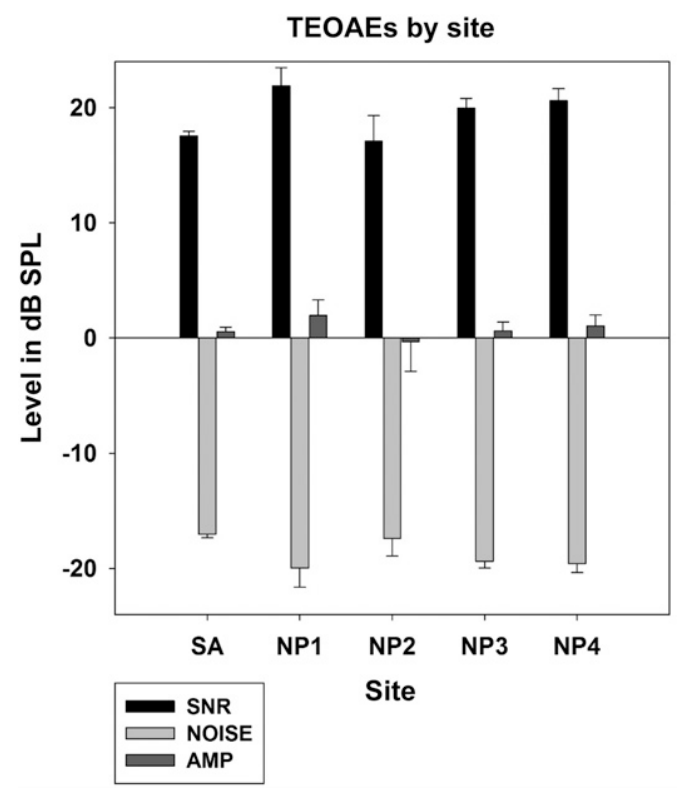

Figure 3. TEOAE measures shown separately by site. The NP5 site is omitted because of the small number of participants.

2006). These studies justified the use of a one-tailed $t$-test for both TEOAE and ABR for testing the hypothesis of a right ear advantage.

\section{RESULTS}

\section{TEOAE}

Average TEOAEs were initially inspected by ear and age at assessment (newborn and 1 month). TEOAE SNR results are shown for each ear graphically in Figure 1. Adapting a convention used when TEOAEs are administered in a screening protocol, a subset of infants was identified with responses having SNRs of $4 \mathrm{~dB}$ or greater in four of the six frequency bands in both ears. These responses were termed a "bilateral pass." This category included 241 assessments from 168 participants which are broken down by age at test, sex, and test site in Table 3 . When a baby was tested twice, either or both tests could be considered a "bilateral pass" regardless of the outcome of the other test. These "bilateral pass" test results presume that the infant was, at the time of the test, free from common transient contaminations of the TEOAE such as ear canal debris or middle ear effusions. These infants were also considered to have outer hair cell function that is within normal limits.
Figure 1 shows TEOAE SNR by frequency band and for overall wideband response in the right and left ears. The data from the subset of infants in the "bilateral pass" category and those in the "bilateral fail" category levels are shown separately from the entire group. Newborn responses are depicted with open symbols and infants at 1 month of age are shown with filled symbols

Tables 4 and 6 show the ANOVA results for evaluation of factors potentially affecting overall TEOAE SNRs including age, sex, and test site. Test site was chosen rather than maternal race because of the high correlation between the two and the fact that when both are evaluated, only test site is significant. Some infants were tested more than once (at birth and 1 month) and, therefore, the number of tests was used as a covariate. The SNRs from the right and left ears were combined to create the TEOAE SNR Factor. Table 4 shows the analysis using all participants whereas Table 6 shows the ANOVA results using only the "bilateral pass" infants. Table 5 gives the significance results of post hoc comparisons of TEOAE SNR by site. The SA site was found to have significantly lower SNR than three of the four NP sites. With the exception of the influence of infant's age (newborn versus 1 month), which is significant when all participants are included, the conclusions drawn from the two analyses are the same and will be presented in the following paragraphs by the factors involved. No interactions were found between or among age, site, and/or sex.

\section{Age}

A clear developmental trend can be seen in Figure 1. The SNR of the responses grows significantly ( $p=$ 0.004 ) between the newborn period and the first month of life in the overall group. The overall SNR of all babies grew by 4.54 and $3.12 \mathrm{~dB}$ in the right and left ears, respectively. ANOVA with repeated measures on the frequency-specific TEOAE bands for "bilateral pass" participants, after averaging left and right ears, demonstrated main effects for frequency $(p<0.001)$ and age $(p<0.001)$ and an interaction between age and frequency $(p<0.001)$.

To further investigate changes in the TEOAE SNR between the newborn and the first month of life, changes in TEOAE frequency-specific amplitude and background noise measures are plotted separately as a function of age in Figure 2. TEOAE amplitude is

Table 7. Potential Association of Pass-Fail Status with Test Site Based on the Chi-Squared Test of Independence $\left(\chi^{2}=16.203, \mathrm{df}=5, p=0.006\right)$

\begin{tabular}{lcccccc}
\hline Test Site & SA & NP1 & NP2 & NP3 & NP4 & Total \\
\hline Pass & $154 / 0.52$ & $16 / 0.84$ & $7 / 0.58$ & $37 / 0.69$ & $24 / 0.75$ & $238 / 0.57$ \\
Fail & $143 / 0.48$ & $3 / 0.16$ & $5 / 0.42$ & $17 / 0.31$ & $8 / 0.25$ & $176 / 0.43$ \\
Total & 297 & 19 & 12 & 54 & 32 & 414 \\
\hline
\end{tabular}


shown to increase with age $(p<0.001)$ but noise does not change ( $p=0.99)$.

\section{Testing Site}

Six test sites were represented in the data as shown in Table 2. Test site was found to have a significant influence on TEOAE SNR $(p<0.001$, Tables 4-6).

Figure 3 illustrates the TEOAE test results from five of the six sites (NP5 with only three cases was omitted). The SA and NP2 sites show the lowest SNRs. Post hoc analysis (Table 5) found that the SA site SNR was significantly lower than other sites with the exception of NP2. ANOVAs were performed for TEOAE SNR, amplitude, and noise. Only the TEOAE noise was significantly different by site ( $p=0.006)$ with SA having higher noise than NP1, NP3, and NP4 on post hoc analysis.

\section{Pass-Fail Analysis}

Factors affecting bilateral pass status, including test site, age, and sex were evaluated using the chi-square test for independence; the results can be found in Tables $7-9$. Neither age nor sex showed any influence on pass status. Only test site was significant for differences among the pass-fail categories. The results range from $52 \%$ pass at SA to $84 \%$ pass at NP1, indicating a wide range of results by site.

\section{Ear}

Comparisons of left versus right ear for TEOAE amplitude, background noise, and SNR are shown in Figure 4. The amplitude $(p<0.001)$ and background noise $(p=$ 0.006 ) of the responses from the right ear are significantly larger than those from the left, but the resulting SNRs do not differ between ears. A separate plot of the TEOAE amplitudes (Figure 5) from the bilateral pass infants also shows consistently larger amplitude from the right ear for all frequency bands.

To examine factors influencing amplitude asymmetry, a variable was created (amplitude asymmetry R-L) by subtracting the left amplitude from the right for the bilateral pass participants. This variable was analyzed by ANOVA with age, sex, and maternal race as factors. None of these factors had significant influence on laterality in this sample, but there was an interaction between sex and age $(p=0.003)$.

\section{ABR}

An example of an $A B R$ recording from a newborn infant is shown in Figure 6. Each recording is the result of the addition of two individual recordings each comprised of an average responses to 1,024 stimuli after
Table 8. Potential Association of Pass-Fail Status with Age Based on the Chi-Squared Test of Independence $\left(\chi^{2}=5.04, \mathrm{df}=1, p=0.14\right)$

\begin{tabular}{lcccc}
\hline Age & 1 Month & Newborn & Total & $p=0.14$ \\
\hline Pass & $55 / 0.68$ & $113 / 0.53$ & $168 / 0.57$ & \\
Fail & $26 / 0.32$ & $102 / 0.47$ & $128 / 0.43$ & \\
Total & 81 & 215 & 296 & \\
\hline
\end{tabular}

noisy artifacts have been discarded. The response from an infant typically contains at least three peaks or waves that are referred to as I, III, and V.

Several factors were evaluated relative to ABR peak latency and amplitude using ANOVAs with repeated measures. Between-subject factors included maternal race, infant's age, and sex. For the TEOAE analysis, the test site was found to be a significant factor because of the higher levels of acoustic noise present at some sites. In contrast, the ABR is relatively unaffected by acoustic noise, but the amplitude of the ABR could be affected by electrical noise in the environment. However, initial analyses showed no influence of test site on ABR amplitude ( $p=0.55$ ). Therefore, maternal race was used in the analysis rather than test site. The results of the two ANOVAs are shown in Tables 10 and 11. ABR amplitude increased significantly with age $(p<0.001)$, whereas the latency decrease over the first month was close but did not reach significance $(p=0.058)$. Neither maternal race nor sex was found to influence ABR measures from this population, and no interactions were found.

\section{Age}

Figure 7 shows $A B R$ peak latency and amplitude as a function of age. The amplitudes of ABR peaks are larger and latencies shorter at the older (1 month) age compared with the newborn. In this instance, the latency difference approaches but does not achieve statistical significance $(p=0.06)$, whereas the amplitude differences are significant $(p<0.001)$.

\section{Sex}

Male and female ABR indices are shown in Figure 8. The female responses appear larger and earlier than the male responses, but these differences did not achieve significance.

Table 9. Potential Association of Pass-Fail Status with Sex Based on the Chi-Squared Test of Independence $\left(x^{2}=0.09, \mathrm{df}=1, p=0.76\right)$

\begin{tabular}{llccc}
\hline Sex & Female & Male & Total & $p=0.76$ \\
\hline Pass & $93 / 0.56$ & $75 / 0.58$ & $168 / 0.57$ & \\
Fail & $74 / 0.44$ & 54.42 & $128 / 0.43$ & \\
Total & 167 & 129 & 296 & \\
\hline
\end{tabular}



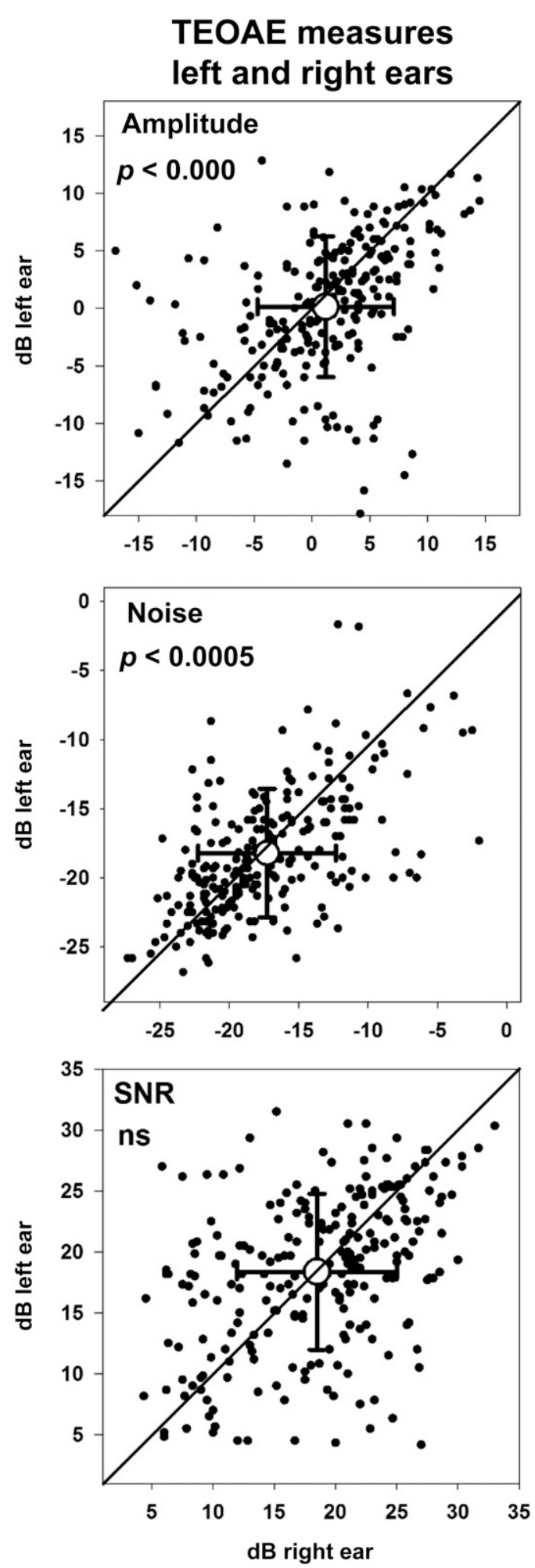

Figure 4. Left-right ear comparisons for TEOAE background noise, amplitude, and SNR for bilateral pass participants. Scatter plots of data from left versus right ear are shown with superimposed mean values and error bars indicating \pm 1 standard deviation. Both noise and amplitude are statistically larger in the right compared with the left ear, but SNRs are balanced across left and right ears (symmetrical).

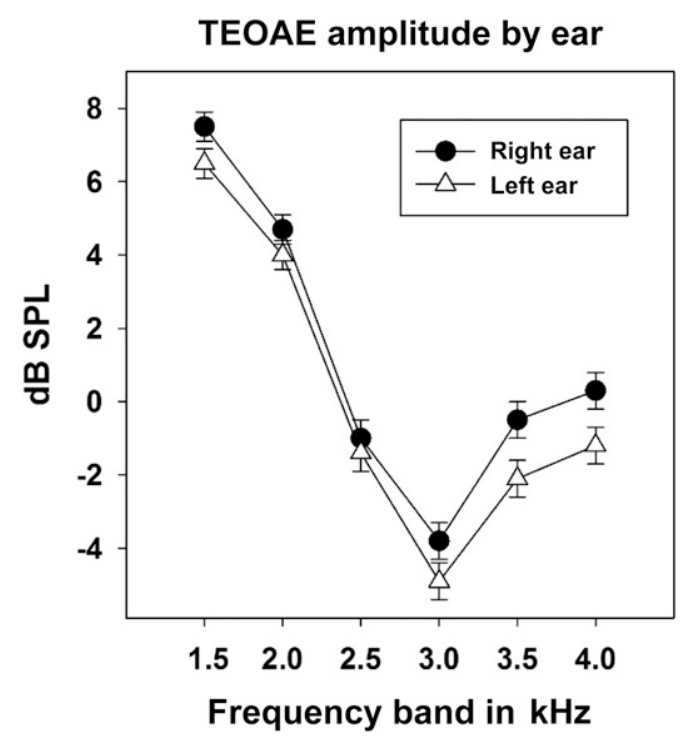

Figure 5. TEOAE amplitude by frequency band demonstrates consistently larger values for the right ear. Only infants in the "bilateral pass" group are included.

\section{Maternal Race}

Latency-by-amplitude plots for all ABR peaks by maternal race categories are shown in Figure 9. Neither amplitude nor latency was significantly influenced by race.

\section{Left Versus Right Ear}

Figure 10 plots the latencies by amplitude of the three major peaks for left and right ears. Latency/amplitude pairs were available for 236 cases and paired $t$-tests found shorter latencies from the right ear with $p=0.003, p=0.014$, and $p=0.019$ for waves I, III, and $\mathrm{V}$, respectively. In contrast, peak amplitudes $(\mathrm{N}=235)$ did not differ significantly by ear for any of the three peaks. Also compared were the interpeak latencies between waves I and V. No significant difference in I-V latency interval was found for left versus right ears.

Interpeak Latencies and Amplitudes: Interpeak I-V latencies and V/I amplitude ratios are shown by Ear, Gender, Age, and race on Table 12. Paired $t$-tests found that neither amplitude ratios $(p=0.250)$ nor latency intervals $(p=0.097)$ were distinguished by ear. None of the other factors was found to have significant effect on latency interval or amplitude ratio, and no interactions were found.

Combined TEOAE and ABR Measures: To investigate whether a combination of TEOAE and ABR results can be used to predict which infants may have middle ear fluid, the ABR data were evaluated relative to TEOAE status (pass or fail) based on the criteria explained 


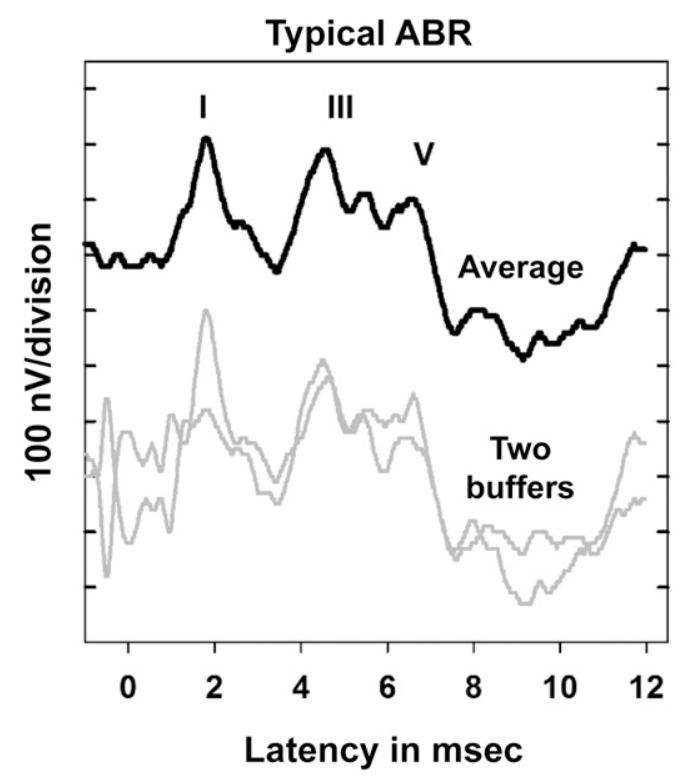

Figure 6. Example of a newborn $A B R$ recording. An ABR recording from a typical newborn is shown at the top. Each ABR is the average of two buffers shown below, one elicited with a condensation and the other with a rarefaction click stimulus. Buffers are comprised of 1,024 sweeps each and added to create the final average. The early peaks of the buffers $(<2 \mathrm{msec})$ are out of phase and constitute the cochlear microphonic (Starr et al, 2001). Stimuli used were $80 \mathrm{~dB}$ nHL clicks presented at $13 /$ sec. Response peaks are labeled conventionally as Waves I, III, and V. Peaks are defined by their poststimulus latency in msec and amplitude from peak to the following trough in $\mu \mathrm{V}$.

previously. ABR peak latency and amplitude for Waves $\mathrm{I}$ and $\mathrm{V}$ from the left and right ears of newborns were compared based on the pass or fail outcome of the TEOAE test performed at the same time. The results are depicted in Figure 11 and given in Table 13.

Independent sample $t$-tests were used to compare the latencies and amplitudes of Waves I and V in the "pass" TEOAE condition compared with the "fail" TEOAE condition. Significant differences based on the pass/fail condition were found only for the right ear; for Wave V Latency $(p=0.023)$ and for Wave I Amplitude $(p=0.049)$.

\section{DISCUSSION}

$\mathrm{T}$ his report describes the auditory system data acquired from a subsample of the Safe Passage Study infants who were not exposed to maternal alcohol drinking or cigarette smoking or drugs of abuse prenatally and who were born at term gestation with normal birth weight. The results demonstrate the validity of the methods used and quality of data acquired and supports the proposed application of these measures for investigating effects of prenatal exposures on auditory system function.

\section{TEOAE}

Lateral asymmetry has been noted in TEOAEs of infants with right ear SNR sometimes found to be larger than left (Aidan et al, 1997; Khalfa et al, 1997; Thornton et al, 2003; Saitoh et al, 2006; Sininger and ConeWesson, 2006). This right-ear advantage for OAEs is reflected in newborn hearing screening outcomes as well (Ari-Even Roth et al, 2016). An important aspect of this study was the use of a standard number of stimulus presentations in the acquisition of the TEOAE. Whereas screening for auditory dysfunction with TEOAEs may be more efficient when data acquisition stops as soon as an acceptable SNR is reached, the screening approach allows the number of stimuli to vary for each acquisition. Averaging for both the $\mathrm{ABR}$ and the TEOAE is the process that reduces the noise to reveal the response, if present. The noise is reduced exponentially with the number of averages (Don and Elberling, 1994). The measurement of the response amplitude is always influenced by the amount of background noise (Backus, 2007). By fixing the number of sweeps acquired, and setting that number high enough to expect adequate reduction of background noise, the response amplitude and noise (and consequently the SNR) can be compared across conditions (Sininger and Cone, 2008). In other studies, this is not the case (Berninger, 2007; Prieve et al, 2009). Clearly SNR can be driven by response amplitude and/or noise, but until all are measured

Table 10. Repeated Measures Analysis of Variance (ANOVA) for Factors with Potentially Significant Influence on ABR Peak Amplitude

\begin{tabular}{lccccc}
\hline \multicolumn{4}{c}{ ANOVA-Dependent Variable: ABR Peak Amplitude } & & \\
\hline Source & Type III Sum of Squares & df & Mean Square & $F$ & Significance, $p$ Value \\
\hline Intercept & 13.73 & 1 & 13.73 & 415.79 & $<0.001$ \\
Sex & 0.07 & 1 & 0.07 & 2.12 & 0.15 \\
Maternal race & 0.16 & 2 & 0.08 & 2.41 & 0.09 \\
Age & 0.80 & 1 & 0.80 & 24.22 & 0.001 \\
Sex * Maternal race & 0.003 & 2 & 0.002 & 0.05 & 0.33 \\
Sex * Age & 0.01 & 1 & 0.01 & 0.23 & 0.63 \\
Maternal race * Age & 0.008 & 1 & 0.008 & 0.42 & 0.52 \\
Sex * Maternal race * Age & 0.01 & 224 & 0.01 & 0.03 & \\
Error & 7.40 & & &
\end{tabular}


Table 11. Repeated Measures ANOVA for Factors with Potentially Significant Influence on ABR Peak Latency

\begin{tabular}{lccccc}
\hline \multicolumn{4}{c}{ ANOVA-Dependent Variable: ABR Peak Latency } & & \\
\hline Source & Type III Sum of Squares & df & Mean Square & $F$ & Significance, $p$ Value \\
\hline Intercept & $8,894.52$ & 1 & $8,894.52$ & $6,811.79$ & $<0.001$ \\
AGE & 4.76 & 1 & 4.76 & 3.64 & 0.06 \\
Sex & 0.02 & 1 & 0.02 & 0.01 & 0.91 \\
Maternal race & 2.03 & 2 & 1.02 & 0.78 & 0.46 \\
Age * Sex & 0.15 & 1 & 0.15 & 0.11 & 0.74 \\
Age * Maternal race & 0.62 & 1 & 0.62 & 0.48 & 0.49 \\
Sex * Maternal race & 3.18 & 2 & 1.59 & 1.22 & 0.30 \\
Age * Sex * Maternal race & 0.58 & 226 & 0.58 & 0.44 & 0.51 \\
Error & 295.10 & 1.31 & &
\end{tabular}

independently, the source of the change cannot be determined.

A significant effect of frequency on TEOAE SNR for the average combined left and right ears of "bilateral pass" participants was noted. Figure 1 demonstrates that the general pattern of the greatest contributions to the overall TEOAE response comes from the 2.5 to $4 \mathrm{kHz}$ regions, as has been shown by Berninger (2007). This trend appears to be more pronounced in the 1-month-old infants than in the newborns, and this is supported by the significant frequency-by-age interaction $(p<0.001)$.

An increase in TEOAE SNR was demonstrated over the first month of life as has been noted by Prieve et al (2009). Norton et al (2000) also showed an increase in the $4 \mathrm{kHz}$ band of the TEOAE amplitude up to about 6 weeks chronological age. When left and right ears are averaged, participants in the current study demonstrated a growth in the SNR of $3.83 \mathrm{~dB}$ in the first month and the participants with "bilateral pass" showed growth of $3.04 \mathrm{~dB}$. Prieve et al (2009) found an average $3.3 \mathrm{~dB}$ change in overall amplitude of infants with a "pass" status over the same 1-month period. In contrast, Abdala et al (2008) state that there was "little change in amplitude" from birth to 6 months of age in the distortion-product otoacoustic emission. Their database and analysis focused on postnatal ages of $3,4,5$, and 6 months in a longitudinal design. However, inspection of their Figure 3 that includes time points of term and 4 weeks seems to show a substantial rise in distortion-product otoacoustic emission amplitude over the first month of life from a cross-sectional database.

New information regarding the change in TEOAE SNR between birth and 1 month of age is offered in Figure 2 , which shows that the increase in SNR can be attributed to a growth in TEOAE amplitude (3.76 dB, $p<$ 0.001 ), rather than to a reduction in noise, which demonstrated an insignificant ( $p=0.993)$ change of $-0.1 \mathrm{~dB}$ between the newborn and 1-month time period. This increase in TEOAE amplitude that continues probably up to 6 weeks of age (Norton et al, 2000) is counter to what would be expected based on the growth in the ear canal volume over the first month. This volume growth would be reflected in a reduction of TEOAE, if the otoacoustic emissions did not change over time. The amplitude increase in TEOAE must be sufficient to overcome the volume change. It is possible that at 6 weeks of age and older the change in canal volume is greater than or equal to any potential increase in TEOAE and the net effect is to show no growth.

Test site was found to have a significant effect on TEOAE SNR (Tables 4 and 6). Only noise, but not amplitude, was found to differ across sites. The SA site, located in Cape Town, South Africa, was found to have the highest background noise levels and the lowest "pass" rate for TEOAE. High levels of ambient background noise have long been known to influence outcomes on TEOAE screening (Jacobson and Jacobson, 1994; Headley et al, 2000) and hospitals in Sub-Saharan Africa have been noted to have concerns related to potentially high ambient noise levels and the consequential effects on TEOAE outcomes (Olusanya, 2010). Whereas TEOAE

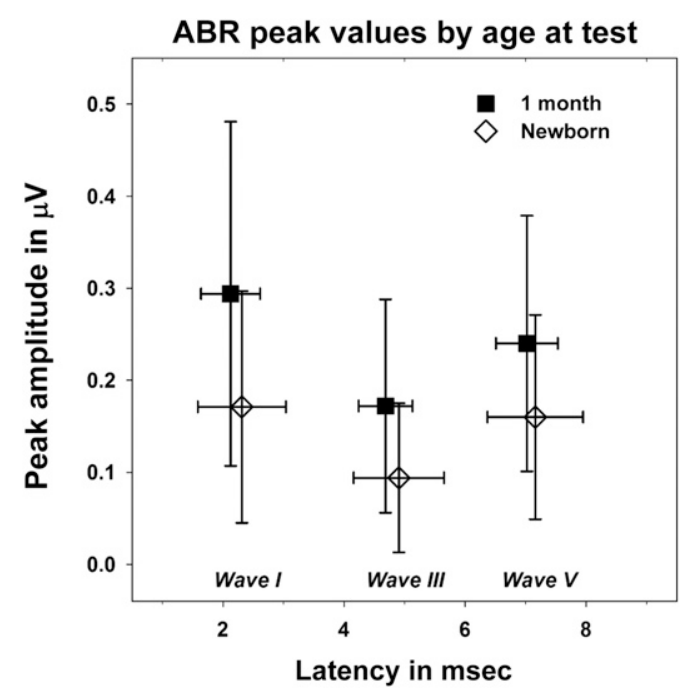

Figure 7. Display of mean peak amplitudes by latency for ABR tests performed in the newborn period and at 1 month of age. As expected, latencies are shorter, and amplitudes are larger at 1 month compared with the newborn, but only the amplitude changes achieve statistical significance (see Tables 10 and 11). Error bars indicate \pm 1 standard deviation. 


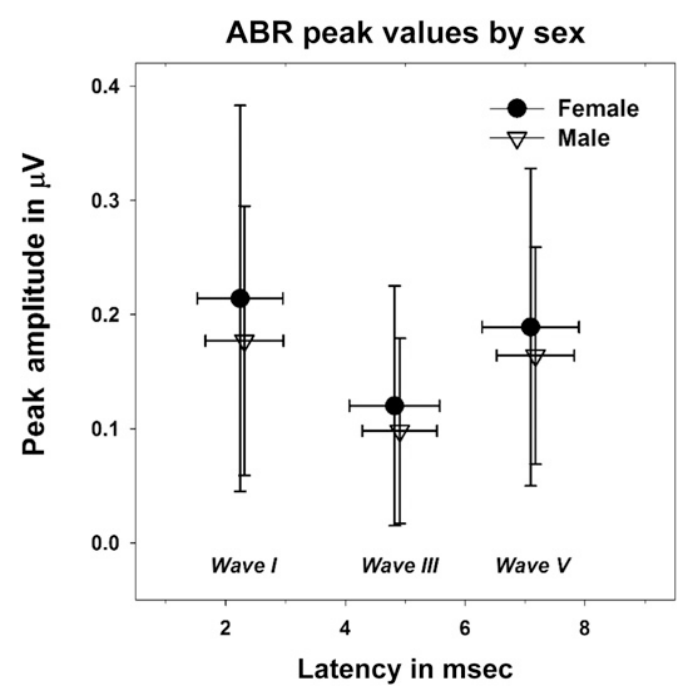

Figure 8. Display of mean peak amplitude by ABR latencies for Wave I, II, and III for male and female infants. Although mean amplitudes are greater for females and latencies are slightly shorter, these differences did not reach statistical significance based on repeated measures ANOVA (see Tables 10 and 11). Error bars indicate \pm 1 standard deviation.

background noise levels are highest at the South Africa site, it is important to note that the test equipment will reject averages that are judged to be "too noisy" and if noise levels are excessive, the test will not be complete. Therefore, only TEOAEs that were had overall noise levels below rejection levels were included in this data set and used in the analysis.

Another factor that distinguished the SA site was found in the test protocol. Because of early discharge, infants from the SA site were brought back for testing

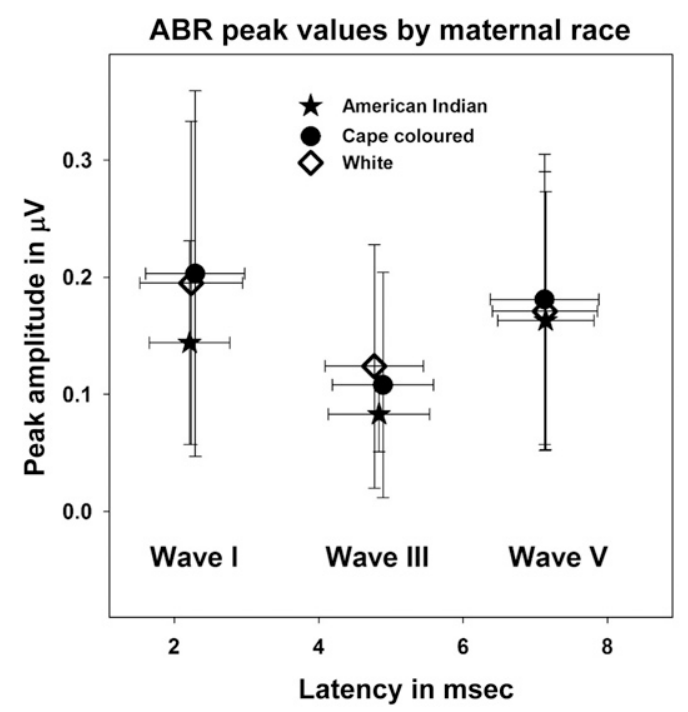

Figure 9. Display of ABR mean peak amplitude by latency for infants by maternal race. No significant effect of maternal race was found for latency or amplitude (see Tables 10 and 11). Error bars indicate $\pm 1 \mathrm{SD}$.

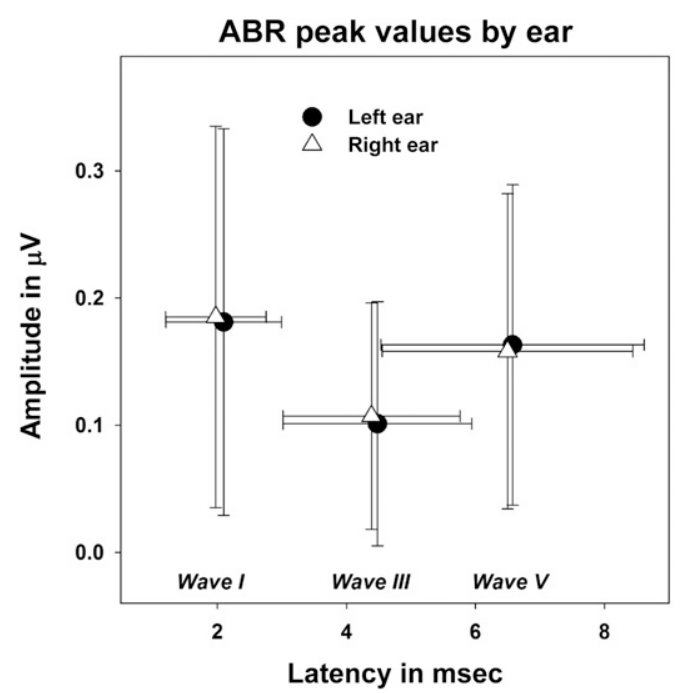

Figure 10. Display of $A B R$ mean peak amplitude by latency for the left and right ears. Error bars indicate \pm 1 standard deviation. The latencies in the right ears are significantly shorter for peaks I $(p=0.003)$, III $(p=0.014)$, and V ( $p=0.019)$; however, amplitudes did not differ by ear (see text).

between 48 and 96 hours after birth. It is possible that the infants being slightly older (2-4 days) were less likely to sleep as well as a 1-2-day-old neonate, raising the fail rates.

The right ear advantage for TEOAE SNR has been noted in many studies (Aidan et al, 1997; Kei et al, 1997; Khalfa et al, 1997; Thornton et al, 2003; Sininger and Cone-Wesson, 2004; Berninger, 2007), but not all. Prieve et al (2009) found no ear differences. Likewise, lateral asymmetry (ear differences) for TEOAE $S N R$ was not statistically significant in this sample of participants. However, the TEOAE amplitude was noted to be larger in the right ear than the left as was demonstrated on Figures 4 and 5.

The fact that ear differences in OAEs are subtle and require a large sample size to be revealed (Berninger, 2007) makes the phenomenon no less real. Those studies revealing a significant right ear advantage for TEOAE SNR included Kei et al (1997) with 567 infants, Sininger and Cone-Wesson (2004) with data from 1,593 infants, Berninger (2007) with 21,560, and Thornton et al (2003) analyzing 28,393 infants. Studies with smaller sample sizes may fail to find lateral asymmetry such as Prieve et al (2009) with 79 participants and the current study which found partial evidence with 168 participants.

\section{ABR}

There are three prominent peaks in an infant's ABR in response to moderate-high-level stimuli, Waves I, III, and $\mathrm{V}$, which are labeled on the average (see Figure 6 for a typical ABR recording).

Some, but not all, of the expected right ear advantages (shorter latencies and larger amplitudes) were 
Table 12. Left (LE) and Right Ear (RE) Wave I to V Latency Intervals and Wave V/I Amplitude Ratios by Sex, Age, and Maternal Race

\begin{tabular}{|c|c|c|c|c|c|c|c|c|c|}
\hline & & \multicolumn{2}{|c|}{ LE I-V Latency } & \multicolumn{2}{|c|}{ RE I-V Latency } & \multicolumn{2}{|c|}{ LE V/I Amp Ratio } & \multicolumn{2}{|c|}{ RE V/l Amp Ratio } \\
\hline & & Mean & $\begin{array}{l}\text { Standard } \\
\text { Deviation }\end{array}$ & Mean & $\begin{array}{l}\text { Standard } \\
\text { Deviation }\end{array}$ & Mean & $\begin{array}{l}\text { Standard } \\
\text { Deviation }\end{array}$ & Mean & $\begin{array}{l}\text { Standard } \\
\text { Deviation }\end{array}$ \\
\hline$\overline{\text { All }}$ & & 4.859 & 0.429 & 4.893 & 0.441 & 1.796 & 3.688 & 1.463 & 2.359 \\
\hline \multirow[t]{2}{*}{ Infant sex } & Female & 4.852 & 0.464 & 4.870 & 0.447 & 1.76 & 3.68 & 1.58 & 2.81 \\
\hline & Male & 4.866 & 0.385 & 4.918 & 0.423 & 1.74 & 3.51 & 1.36 & 1.50 \\
\hline \multirow[t]{2}{*}{ Age } & 1 month & 4.900 & 0.420 & 4.886 & 0.410 & 1.32 & 0.72 & 1.11 & 1.19 \\
\hline & Newborn & 4.847 & 0.432 & 4.892 & 0.445 & 1.87 & 4.04 & 1.58 & 2.54 \\
\hline \multirow[t]{3}{*}{ Maternal race } & $\begin{array}{c}\text { American Indian or } \\
\text { Alaska Native }\end{array}$ & 4.931 & 0.340 & 5.055 & 0.341 & 1.26 & 1.18 & 1.47 & 1.69 \\
\hline & $\begin{array}{l}\text { South African } \\
\quad \text { (Mixed ancestry) }\end{array}$ & 4.839 & 0.449 & 4.851 & 0.426 & 1.88 & 4.20 & 1.66 & 2.63 \\
\hline & White & 4.898 & 0.387 & 4.975 & 0.482 & 1.49 & 1.24 & 0.89 & 0.78 \\
\hline
\end{tabular}

Note: None of these factors had significant influence on latency intervals or amplitude ratio and no interactions were found on repeated measures ANOVA. Ear comparisons were nonsignificant for $\mathrm{V} / \mathrm{I}$ amplitude ratio $(p=0.250)$ and I-V Latency Interval $(p=0.097)$.

found in this data set. The latencies of all peaks were found to be significantly shorter for right ear stimulation but the amplitudes, which have much greater variability, were not significantly different across ears. Sininger and Cone-Wesson (2006) looked at these variables with respect to ear asymmetry in a much larger sample $(>2,000)$ of newborns with a $69 \mathrm{~dB}$ click stimulus. They found that the latencies of waves III and V were shorter from the right ear but found a reversal for wave I (left ear generated latency was shorter than the right). Also unlike this study, Sininger and ConeWesson found that the amplitude of Wave $\mathrm{V}$ from the right ear was significantly larger than from the left. As with TEOAEs, the discrepancies in findings between this study and previous ones may be indicative of the smaller sample size and slight differences in protocol.

It has been well documented that the ABR latencies diminish and amplitudes increase over the first 2-to-3 years of life (Salamy, 1984). Our measurement time interval is short, only 1 month in duration, and yet the increase in response amplitude is apparent and the latency decreases in the expected direction as displayed on Figure 7.

Female participants are often shown to have larger amplitude and earlier latency ABR peaks, likely because of anatomical differences in their cochleae relative to males (Don et al, 1994). Sex differences in ABR peaks are suggested by the earlier latencies and larger amplitudes of the female responses demonstrated in Figure 8. However, these trends did not achieve statistical significance in our sample.

None of the factors tested (ear, sex, age, or maternal race) was found to influence either the I-V latency interval or the V/I amplitude ratio of the infants in this study. Eggermont and Salamy (1988) and others have shown that the I-V latency interval does decrease over the first 2 years of life. The short 1-month interval evaluated in this study may not have allowed sufficient time to see this trend. Sininger and Cone-Wesson (2006) also found no ear differences in the I-V latency interval.

\section{ABR with TEOAE Status}

The changes in Wave I and $\mathrm{V}$ amplitudes and latencies with TEOAE pass/fail status (Table 13 and Figure 11) are somewhat inconsistent. Significant changes in ABR

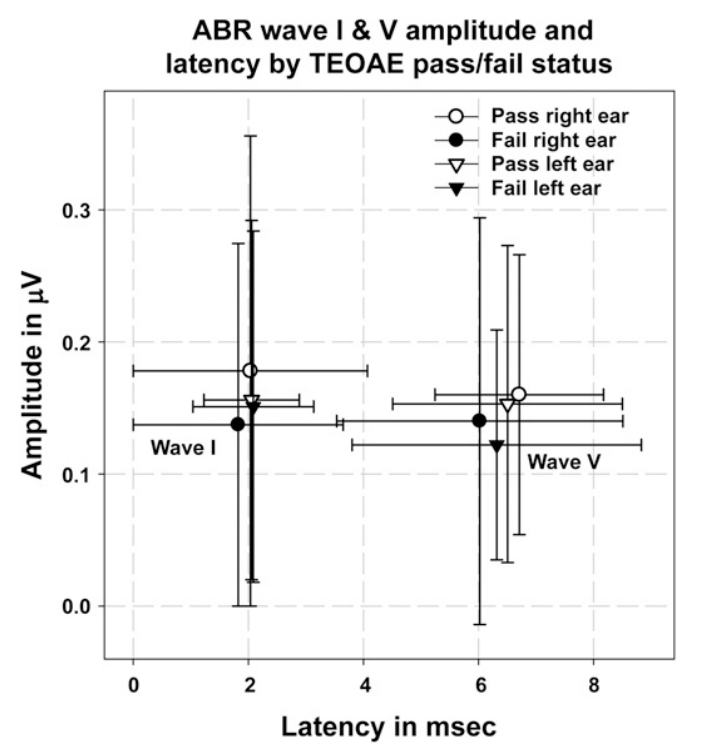

Figure 11. ABR Waves I and V compared by infants' TEOAE pass/fail status (see text for definition) to investigate whether TEOAE status is reflected in ABR latencies or amplitudes. Bivariate displays of ABR Wave $\mathrm{V}$ amplitude versus latency are shown for participants who "passed" the TEOAE (open symbols) and those whose response was recorded as a "fail" (closed). The data are further divided by right versus left ear. Significant differences (see Table 13) were found for the Wave V latency by TEOAE status in the right ear $(p=0.023)$ and for Wave I amplitude by status also in the right ear $(p=0.049)$. 
Table 13. Latency and Amplitude Data for ABR Waves I and V Based on Pass/Fail Condition for the TEOAE

\begin{tabular}{|c|c|c|c|c|c|c|c|c|c|c|c|c|c|}
\hline & & \multicolumn{3}{|c|}{ Wave V Latency } & \multicolumn{3}{|c|}{ Wave V Amplitude } & \multicolumn{3}{|c|}{ Wave I Latency } & \multicolumn{3}{|c|}{ Wave I Amplitude } \\
\hline & & Mean & $\begin{array}{l}\text { Standard } \\
\text { Deviation }\end{array}$ & $\begin{array}{l}t \text { test } \\
p=\end{array}$ & Mean & $\begin{array}{l}\text { Standard } \\
\text { Deviation }\end{array}$ & $\begin{array}{l}t \text { test } \\
p=\end{array}$ & Mean & $\begin{array}{l}\text { Standard } \\
\text { Deviation }\end{array}$ & $\begin{array}{l}t \text { test } \\
p=\end{array}$ & Mean & $\begin{array}{l}\text { Standard } \\
\text { Deviation }\end{array}$ & $\begin{array}{c}t \text { test } \\
p=\end{array}$ \\
\hline \multirow[t]{3}{*}{ Left ear } & All & 6.814 & 1.804 & & 0.199 & 0.143 & & 2.158 & 0.907 & & 0.233 & 0.171 & \\
\hline & Fail & 6.316 & 2.515 & 0.601 & 0.122 & 0.087 & 0.056 & 2.085 & 1.049 & 0.843 & 0.151 & 0.133 & 0.814 \\
\hline & Pass & 6.505 & 1.996 & & 0.153 & 0.120 & & 2.056 & 0.828 & & 0.156 & 0.136 & \\
\hline \multirow[t]{3}{*}{ Right ear } & All & 6.574 & 1.983 & & 0.168 & 0.120 & & 2.004 & 0.775 & & 0.225 & 0.171 & \\
\hline & Fail & 6.021 & 2.487 & 0.023 & 0.140 & 0.154 & 0.319 & 1.823 & 0.865 & 0.088 & 0.137 & 0.127 & 0.049 \\
\hline & Pass & 6.708 & 1.464 & & 0.160 & 0.106 & & 2.035 & 0.731 & & 0.178 & 0.135 & \\
\hline
\end{tabular}

metrics based on TEOAE status were only seen in the ABRs from the right ear. Amplitude was significantly smaller in the TEOAE fail condition but only for Wave I, and latency was significantly shorter only for Wave V. Shorter latencies would not be consistent with the presence of a conductive block and yet three of four latencies compared were shorter in TEOAE fail condition. These findings do not support the idea that the reason for a TEOAE failure was reflected in the ABR measures. The most common cause of TEOAE "fail" in the newborn period is some type of conductive block in the middle or outer ear (Thornton et al, 1993; Doyle et al, 1997; Keefe et al, 2003). The same reason for failure on TEOAE must be assumed for some of this study's population. However, the ABR stimulus used in this study was a high-level, $80 \mathrm{~dB}$ nHL. Whereas a mild conductive block may obliterate an otoacoustic emission, the same would create only a mild attenuation of the sound level of the ABR-eliciting stimulus and have a negligible effect on the ABR latency or amplitude.

We conclude that the auditory system data acquired for the Safe Passage Study, as shown in the analyses from this subsample of "unexposed" infants, is consistent with reports of these auditory system measures, TEOAEs, and $\mathrm{ABRs}$, in the general population. The results are consistent with the literature on newborn auditory system measures and reflect a high standard of data acquisition. Hence, these auditory system measures can be informative with respect to the hypotheses that prompted initiation of the PASS Network study and, therefore, be used in subsequent analysis of exposure variables and other risk factors ascertained in this large cohort study.

Acknowledgments. Research reported in this publication was supported by National Institutes of Health grants U01HD055154, U01HD045935, U01HD055155, U01HD045991, and U01AA016501 funded by the National Institute on Alcohol Abuse and Alcoholism, Eunice Kennedy Shriver National Institute of Child Health and Human Development, and the National Institute on Deafness and Other Communication Disorders.

The PASS Network is solely responsible for the design and conduct of the study; collection, management, analysis, and interpretation of the data; and preparation, review, or approval of the manuscript. The following researchers composed the PASS Network:
- PASS Steering Committee Chair (University of Texas Medical Branch): Gary DV Hankins, MD

- Data Coordinating \& Analysis Center (DM-STAT, Inc.): PI: Kimberly A Dukes, PhD; Co-PI: Lisa M Sullivan, $\mathrm{PhD}$; Biostatistics: Tara Tripp, MA; Fay Robinson, MPH; Cheri Raffo, MPH; Project Management/Regulatory Affairs: Julie M Petersen, MPH; Rebecca A Young, MPH; Statistical Programming/Data Management: Cindy Mai, BA; Elena Grillo, MBA, BS, BBA; Data Management/Information Technology: Travis Baker, BS; Patti Folan; Gregory Toland, MS; Michael Carmen, MS

- Developmental Biology \& Pathology Center (Children's Hospital Boston): PI: Hannah C Kinney, MD; Assistant Director: Robin L Haynes, $\mathrm{PhD}$; Co-investigators: Rebecca D Folkerth, MD; Ingrid A Holm, MD; Theonia Boyd, MD; David S Paterson, PhD; Hanno Steen, $\mathrm{PhD}$; Kyriacos Markianos, PhD; Drucilla Roberts, MD; Kevin G Broadbelt, PhD; Richard G Goldstein, MD; Laura L. Nelsen, MD; Jacob Cotton, BS; Perri Jacobs, BS

- Comprehensive Clinical Site Northern Plains (Sanford Research): PI: Amy J Elliott, PhD; Co-PI: Larry Burd, Ph.D.; Co-investigators: Jyoti Angal, MPH; Jessica Gromer, RN; H Eugene Hoyme, MD; Margaret Jackson, BA; Luke Mack, MA; Bradley B Randall, MD; Mary Ann Sens, MD; Deborah Tobacco, MA; Peter Van Eerden, MD

- Comprehensive Clinical Site South Africa (Stellenbosch University): PI: Hendrik Odendaal, MBChB, FRCOG, MD; Co-PI: Colleen Wright, MD, FRCPath, PhD; CoInvestigators: Lut Geerts, MD, MRCOG; Greetje de Jong, MBChB, MMed, MD; Pawel Schubert, FCPath (SA) MMed; Shabbir Wadee, MMed; Johan Dempers, FCFor Path (SA); Elsie Burger, FCFor Path (SA), MMed Forens Path; Janetta Harbron, $\mathrm{PhD}$; Co-investigator \& Project Manager: Coen Groenewald, MBChB, MMed, FCOG, M Comm

- Physiology Assessment Center (Columbia University): Co-PIs: William Fifer, PhD; Michael Myers, PhD; Coinvestigators: Joseph Isler, $\mathrm{PhD}$; Yvonne Sininger, $\mathrm{PhD}$; Project Management: J David Nugent, MA; Carmen Condon, BA; Data Analysis: Margaret C Shair, BA; Tracy Thai, MA 
- NIH Project Scientists: Marian Willinger, $\mathrm{PhD}$ (NICHD); Dale Hereld, MD, PhD (NIAAA); Howard J Hoffman, MA (NIDCD); Chuan-Ming Li, MD, PhD (NIDCD)

- The authors gratefully acknowledge the cooperation of the study participants, PASS investigators and members of the NICHD Advisory and Safety Monitoring Board: Elizabeth Thom, PhD (Chair); The Reverend Phillip Cato, PhD; James W Collins, Jr, MD, MPH; Terry Dwyer, MD, MPH; George Macones, MD; Philip A May, PhD; Richard M Pauli, MD, PhD; Raymond W Redline, MD; and Michael Varner, MD.

- Additional acknowledgments: the following individuals made significant contributions to the research and warrant recognition:

- PAC: Tracy Thai, MA; Joseph J Violaris, BA

- NIDCD: Chuan-Ming Li, MD, PhD, MSPH

\section{REFERENCES}

Abdala C, Oba SI, Ramanathan R. (2008) Changes in the DP-gram during the preterm and early postnatal period. Ear Hear 29(4):512-523.

Aidan D, Lestang P, Avan P, Bonfils P. (1997) Characteristics of transient-evoked otoacoustic emissions (TEOES) in neonates. Acta Otolaryngol 117(1):25-30.

Ari-Even Roth D, Hildesheimer M, Roziner I, Henkin Y. (2016) Evidence for a right-ear advantage in newborn hearing screening results. Trends Hear 20:20.

Backus BC. (2007) Bias due to noise in otoacoustic emission measurements. J Acoust Soc Am 121(3):1588-1603.

Berninger E. (2007) Characteristics of normal newborn transientevoked otoacoustic emissions: ear asymmetries and sex effects. Int J Audiol 46(11):661-669.

Boone RT, Bower CM, Martin PF. (2005) Failed newborn hearing screens as presentation for otitis media with effusion in the newborn population. Int J Pediatr Otorhinolaryngol 69(3):393-397.

Church MW. (1987) Chronic in utero alcohol exposure affects auditory function in rats and in humans. Alcohol 4(4):231-239.

Church MW, Holloway JA. (1984) Effects of prenatal ethanol exposure on the postnatal development of the brainstem auditory evoked potential in the rat. Alcohol Clin Exp Res 8(3):258-265.

Cone-Wesson B. (2005) Prenatal alcohol and cocaine exposure: influences on cognition, speech, language, and hearing. J Commun Disord 38(4):279-302.

Curns AT, Holman RC, Shay DK, Cheek JE, Kaufman SF, Singleton RJ, Anderson LJ. (2002) Outpatient and hospital visits associated with otitis media among American Indian and Alaska native children younger than 5 years. Pediatrics 109(3): E41-E41.

Don M, Elberling C. (1994) Evaluating residual background noise in human auditory brain-stem responses. J Acoust Soc Am $96(5 \mathrm{Pt}$ 1):2746-2757.

Don M, Ponton CW, Eggermont JJ, Masuda A. (1994) Auditory brainstem response (ABR) peak amplitude variability reflects individual differences in cochlear response times. J Acoust Soc Am 96(6):3476-3491.
Doyle KJ, Burggraaff B, Fujikawa S, Kim J, MacArthur CJ. (1997) Neonatal hearing screening with otoscopy, auditory brain stem response, and otoacoustic emissions. Otolaryngol Head Neck Surg 116(6 Pt 1):597-603.

Dukes KA, Burd L, Elliott AJ, Fifer WP, Folkerth RD, Hankins GD, Hereld D, Hoffman HJ, Myers MM, Odendaal HJ, Signore C, Sullivan LM, Willinger M, Wright C, Kinney HC ; PASS Research Network. The safe passage study: design, methods, recruitment, and follow-up approach. Paediatr Perinat Epidemiol 28(5):455-465.

Eggermont JJ, Salamy A. (1988) Development of ABR parameters in a preterm and a term born population. Ear Hear 9(5):283-289.

Eldredge L, Salamy A. (1996) Functional auditory development in preterm and full term infants. Early Hum Dev 45(3):215-228.

Ey JL, Holberg CJ, Aldous MB, Wright AL, Martinez FD, Taussig LM; Group Health Medical Associates. (1995) Passive smoke exposure and otitis media in the first year of life. Pediatrics 95(5):670-677.

Headley GM, Campbell DE, Gravel JS. (2000) Effect of neonatal test environment on recording transient-evoked otoacoustic emissions. Pediatrics 105(6):1279-1285.

Hunter LL, Davey CS, Kohtz A, Daly KA. (2007) Hearing screening and middle ear measures in American Indian infants and toddlers. Int J Pediatr Otorhinolaryngol 71(9):1429-1438.

Jacobson JT, Jacobson CA. (1994) The effects of noise in transient EOAE newborn hearing screening. Int J Pediatr Otorhinolaryngol 29(3):235-248.

Kable JA, Coles CD, Lynch ME, Carroll J. (2009) The impact of maternal smoking on fast auditory brainstem responses. Neurotoxicol Teratol 31(4):216-224.

Keefe DH, Zhao F, Neely ST, Gorga MP, Vohr BR. (2003) Earcanal acoustic admittance and reflectance effects in human neonates. I. Predictions of otoacoustic emission and auditory brainstem responses. J Acoust Soc Am 113(1):389-406.

Kei J, McPherson B, Smyth V, Latham S, Loscher J. (1997) Transient evoked otoacoustic emissions in infants: effects of gender, ear asymmetry and activity status. Audiology 36(2):61-71.

Khalfa S, Morlet T, Micheyl C, Morgon A, Collet L. (1997) Evidence of peripheral hearing asymmetry in humans: clinical implications. Acta Otolaryngol 117(2):192-196.

Norton SJ, Gorga MP, Widen JE, Vohr BR, Folsom RC, Sininger YS, Cone-Wesson B, Fletcher KA. (2000) Identification of neonatal hearing impairment: transient evoked otoacoustic emissions during the perinatal period. Ear Hear 21(5):425-442.

Olusanya BO. (2010) Ambient noise levels and infant hearing screening programs in developing countries: an observational report. Int J Audiol 49(8):535-541.

Prieve BA, Hancur-Bucci CA, Preston JL. (2009) Changes in transient-evoked otoacoustic emissions in the first month of life. Ear Hear 30(3):330-339.

Rubens DD, Vohr BR, Tucker R, O’Neil CA, Chung W. (2008) Newborn oto-acoustic emission hearing screening tests: preliminary evidence for a marker of susceptibility to SIDS. Early Hum Dev 84(4):225-229.

Saitoh Y, Sakoda T, Hazama M, Funakoshi H, Ikeda H, Shibano A, Yajin S, Yoda S, Dake Y, Enomoto T, Kitano H. (2006) Transient evoked otoacoustic emissions in newborn infants: effects of ear asymmetry, gender, and age. J Otolaryngol 35(2):133-138. 
Salamy A. (1984) Maturation of the auditory brainstem response from birth through early childhood. J Clin Neurophysiol 1(3): 293-329.

Sininger Y, Cone B. (2008) Comment on "Ear Asymmetries in middleear, cochlear, and brainstem responses in human infants" [J. Acoust. Soc. Am. 123, 1504-1512]. J Acoust Soc Am 124(3):1401-1403.

Sininger YS, Cone-Wesson B. (2004) Asymmetric cochlear processing mimics hemispheric specialization. Science 305(5690):1581.

Sininger YS, Cone-Wesson B. (2006) Lateral asymmetry in the ABR of neonates: evidence and mechanisms. Hear Res 212(1-2):203-211.

Sininger YS, Cone-Wesson B, Abdala C. (1998) Gender distinctions and lateral asymmetry in the low-level auditory brainstem response of the human neonate. Hear Res 126(1-2):58-66.
Starr A, Sininger Y, Nguyen T, Michalewski HJ, Oba S, Abdala C. (2001) Cochlear receptor (microphonic and summating potentials, otoacoustic emissions) and auditory pathway (auditory brain stem potentials) activity in auditory neuropathy. Ear Hear 22(2):91-99.

Thornton ARD, Kimm L, Kennedy CR, Cafarelli-Dees D. (1993) External- and middle-ear factors affecting evoked otoacoustic emissions in neonates. Br J Audiol 27(5):319-327.

Thornton ARD, Marotta N, Kennedy CR. (2003) The order of testing effect in otoacoustic emissions and its consequences for sex and ear differences in neonates. Hear Res 184(1-2):123-130.

Willinger M, James LS, Catz C. (1991) Defining the sudden infant death syndrome (SIDS): deliberations of an expert panel convened by the National Institute of Child Health and Human Development. Pediatr Pathol 11(5):677-684. 